Reprod. Nutr. Dévelop., 1988, 28 (6 B), 1687-1698

\title{
(Proto)-oncogènes, croissance cellulaire et développement
}

\author{
Dominique MORELLO
}

Unité de Génétique des Mammifères,

Institut Pasteur, 25 rue du Dr Roux, 75015 Paris.

Summary. (Proto)-oncogenes, cell growth and development.

In the past few years, one realizes that number of genes whose products are involved in regulating normal cell growth and development are also capable of inducing malignancy. These genes are called oncogenes and include $1^{\circ}$ the cellular oncogenes (c-onc) or proto-oncogenes and $2^{\circ}$ the viral oncogenes ( $v$-onc) which are most likely derived from $c$-onc and which are the transforming genes of several strains of animal retrovirus. The c-onc are present in the genome of all vertebrate cells and show a remarkable degree of evolutionary conservation, suggesting that they serve essential cellular functions. The analysis of biological properties of both types of oncogenes is of importance to understand the role of proto-oncogenes in normal cell proliferation and/or differentiation and development and to determine how alterations in their structures play a role in malignant transformation. In order to give an overview of the diversity of oncogene activities, several oncogene products involved in growth control, signal transduction, gene expression and development will be described.

\section{Introduction.}

Grâce aux études entreprises aussi bien sur les cellules normales, dont la croissance est finement contrôlée, que sur les cellules tumorales, qui se divisent de façon anarchique, nous comprenons mieux depuis quelques années les mécanismes qui régulent la prolifération et la différenciation cellulaire. En particulier l'étude de la transformation des cellules infectées par des rétrovirus animaux a joué un rôle prépondérant. En effet, plusieurs souches de retrovirus, famille de virus qui possèdent un génome à $A R N$ mais qui se répliquent par l'intermédiaire d'une forme ADN, induisent des tumeurs à progression rapide, fatales pour l'hôte infecté. L'analyse de ces rétrovirus a permis (et permet encore) l'identification de plusieurs dizaines de gènes transformants différents qu'on appelle oncogènes viraux ou $v$-onc, qui sont distincts des gènes requis pour la réplication virale, et qui sont directement responsables de la transformation.

La découverte que la majorité des gènes responsables de la transformation possèdent un équivalent cellulaire dont le produit est impliqué dans les processus normaux de prolifération ou de différenciation cellulaire fut également une étape 
décisive: en 1976, Stéhelin et collaborateurs ont montré que les oncogènes n'étaient pas spécifiques des rétrovirus mais qu'on trouvait des séquences presque identiques dans le génome de toutes les cellules de vertébrés (Stéhelin et al., 1976). Ce sont les oncogènes cellulaires, $c$-onc, ou encore protooncogènes. Lorsqu'elles sont capturées par les rétrovirus, ces séquences se trouvent modifiées dans le génome viral et confèrent aux cellules infectées une capacité de prolifération anormale ; ceci suggère, par contrecoup, que la fonction normale des proto-oncogènes serait de participer à la régulation de la croissance cellulaire.

L'étude de tumeurs non induites par des virus a également permis d'établir une corrélation entre modification de proto-oncogènes et transformation: les premières expériences des groupes de Weinberg et Cooper montraient que I'ADN d'une lignée cellulaire dérivée d'une tumeur de la vessie contenait un gène capable d'induire un phénotype tumorigénique après son transfert dans des cellules cultivées non tumorales (Murray et al., 1981 ; Krontiris et Cooper, 1981). Depuis, l'analyse de nombreuses tumeurs contenant des proto-oncogènes mutés, transloqués (c'est-à-dire qui ont changé de localisation par suite d'un remaniement chromosomique) ou amplifiés a confirmé l'idée selon laquelle l'altération de proto-oncogènes contribuait largement à la transformation maligne.

La première relation entre l'activité biologique d'un oncogène rétroviral et la fonction normale d'un gène cellulaire fut établie à la suite de deux groupes de travaux indépendants : 1) les travaux d'Erickson puis ceux d'Hunter et collaborateurs montraient que certains oncogènes viraux avaient une activité de protéines kinases (Collett et Erickson, 1978), capables en particulier de transférer un groupement phosphate sur les tyrosines de substrats protéiques variés (c'est ce qu'on appelle les tyrosine-kinases) (Hunter et Cooper, 1985). 2) Les travaux du groupe de Cohen sur le facteur de croissance EGF (epidermal growth factor) et son récepteur cellulaire, l'EGF-R, montraient que ce récepteur possédait une activité tyrosine-kinase qui était considérablement accrue après fixation de l'EGF (Ushiro et Cohen, 1980). Ces données suggéraient que la fonction de la tyrosine kinase était de transmettre à l'intérieur de la cellule les signaux mitotiques induits par les facteurs de croissance et que les oncogènes porteurs d'une activité comparable pourraient provoquer une prolifération cellulaire anormale en perturbant d'une certaine manière ces interactions. Cette hypothèse fut vérifiée par la suite avec la découverte que plusieurs oncogènes viraux étaient des versions modifiées de récepteurs à des facteurs de croissance ou même des facteurs de croissance.

Que se passe-t-il lorsqu'une cellule est stimulée par un mitogène ou par un facteur de croissance? Comment les signaux mitotiques sont-ils ultérieurement acheminés au noyau ? Plusieurs équipes ont montré que la toute première réponse cellulaire détectable après stimulation était l'induction dans le noyau de l'expression de deux proto-oncogènes : $c$-myc et $c$-fos (revue dans Müller, 1986). Or des expériences très récentes démontrent que les produits de ces proto-oncogènes interagissent avec des facteurs transcriptionnels et permettent ainsi de moduler l'expression d'un certain nombre de gènes en réponse aux stimuli extracellulaires. Le dessin de la figure 1 représente schématiquement les différentes étapes qui 
aboutissent à la transmission d'un signal du compartiment périplasmique au noyau. Il est actuellement concevable que tout gène codant pour un facteur de croissance (extra-cellulaire), un récepteur (membranaire) à un facteur de croissance, un messager secondaire du signal (cytoplasmique) ou une protéine impliquée dans la régulation de la transcription (nucléaire) soit un protooncogène. Vu la complexité du système, on peut envisager que des altérations à l'une ou l'autre des étapes de la transmission du signal puissent entraîner la dérégulation du contrôle de la croissance et du développement, dont la conséquence est la transformation maligne. L'étude de l'activité d'oncogènes correspondant à chaque maillon du réseau permet de comprendre les mécanismes moléculaires qui régissent les processus de la prolifération et/ou de la différenciation et du développement.

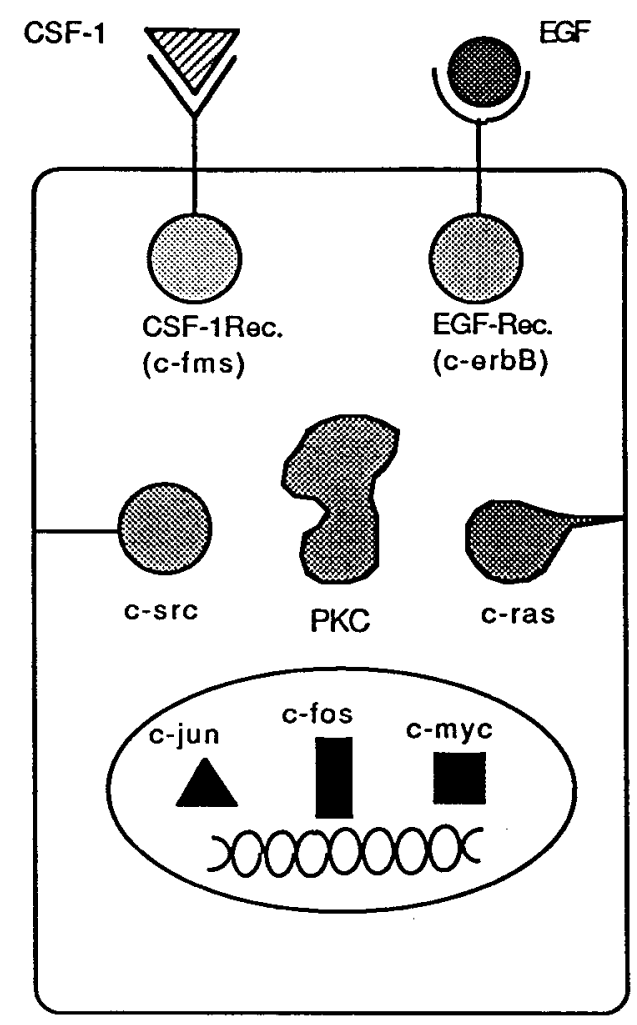

Réception du signal

Transduction du signal

évènements nucléaires

FIG. 1. - Les différentes étapes de la transmission d'un signal mitotique (d'après la couverture du livre "Oncogenes and growth control», Kahn et Graf, 1986).

Nous allons brièvement reprendre chaque étape en l'illustrant par un exemple choisi parmi d'autres. Cette illustration a pour but non d'analyser en détail des 
différents mécanismes impliqués dans ces processus, mais de donner une idée de la diversité fonctionnelle et de la complexité des oncogènes. Les principales caractéristiques des différents produits de proto-oncogènes que nous décrivons sont résumées dans le tableau 1.

\section{TABLEAU 1}

Activité et localisation des produits de quelques proto-oncogènes.

c-onc Identité, homologie ou taille $\quad \begin{gathered}\text { Localisation, } \\ \text { distribution }\end{gathered} \quad$ Activité

$\begin{array}{ll}\text { I. Tyrosine kinase et c-onc apparentés } \\ \text { c-erb-B } & \begin{array}{l}\text { Récepteur EGF } \\ 170 \mathrm{~K}\end{array} \\ \text { C-fms } & \begin{array}{l}\text { Récepteur CSF-1 } \\ 140 \mathrm{~K}\end{array} \\ \end{array}$

II. GTPases

$\begin{array}{llll}\text { C-Ki-ras-2 } & & \text { Partie cytoplasmique de } & \text { Liaison GTP et } \\ \text { C-H-ras-1 } & 21 \mathrm{~K} & \text { la membrane plasmique } & \text { GDP } \\ \mathrm{N} \text {-ras } & & \text { de nombreuses cellules } & \text { GTPase }\end{array}$

\section{Protéines nucléaires}

\begin{tabular}{|c|c|c|c|}
\hline$c-f o s$ & $55 \mathrm{~K}$ & $\begin{array}{l}\text { Noyau de la plupart des } \\
\text { cellules - expression forte } \\
\text { dans les cellules hémato- } \\
\text { poiëtiques, macrophages } \\
\text { et cellules extra embryon- } \\
\text { naires }\end{array}$ & $\begin{array}{l}\text { Liaison indirecte } \\
\text { ADN }\end{array}$ \\
\hline $\begin{array}{l}\mathrm{c}-\mathrm{myc} \\
\mathrm{N}-\mathrm{myc} \\
\mathrm{L}-\mathrm{myc}\end{array}$ & $62-64 \mathrm{~K}$ & $\begin{array}{l}\text { Noyau de la plupart des } \\
\text { cellules }\end{array}$ & Liaison ADN \\
\hline c-jun & AP1 & Noyau & $\begin{array}{l}\text { Liaison } \\
\text { ADN }\end{array}$ \\
\hline
\end{tabular}

\section{Autres}

\begin{tabular}{llll} 
c-erb-A & Récepteur hormone thyroïdienne & $\begin{array}{l}\text { Cytoplasmique et } \\
\text { nucléaire }\end{array}$ & Liaison thyroxine \\
c-sis & Chaîne B du PDGF & Protéine sécrétée & $\begin{array}{l}\text { Liaison récepteur } \\
\text { du PDGF }\end{array}$ \\
\hline
\end{tabular}

(d'après Adamson, 1987).

\section{Oncogènes et contrôle de la croissance.}

L'événement initial de l'action des facteurs de croissance est de se fixer spécifiquement à un récepteur transmembranaire. La liaison du facteur de croissance à son récepteur provoque très probablement l'induction de seconds messagers, intracytoplasmiques et solubles, qui transmettent le signal au noyau. 


\section{Oncogènes et facteurs de croissance.}

C'est en 1983 qu'il fut démontré pour la première fois qu'un oncogène pouvait coder pour une molécule impliquée dans le contrôle de la croissance. Le virus sarcomateux du singe SSV contient l'oncogène $v$-sis qui provoque des cancers variés chez le singe et qui peut également transformer des fibroblastes en culture. Doolittle et al. (1983) et Waterfield et al. (1983) ont montré que la protéine transformante de ce virus, la p28 $8^{\mathrm{v} \text {-sis }}$, était presque identique à la chaîne $\mathrm{B}$ du facteur de croissance PDGF (platelet derived growth factor). Le PDGF est le principal mitogène des cellules dérivées des tissus conjonctifs. II est composé de deux chaînes liées par des ponts disulfures. Son récepteur est une glycoprotéine transmembranaire, dont la région cytoplasmique contient une tyrosinekinase qui est activée par la fixation du ligand. La preuve définitive que le virus SSV avait acquis le gène $v$-sis à partir du gène codant pour la chaîne $B$ fut apportée par le clonage du proto-oncogène cellulaire équivalent du gène $v$-sis : le gène $c$-sis code pour un précurseur de la chaîne $B$. L'analogie entre $v$-sis et le PDGF n'est pas seulement structurale mais aussi fonctionnelle : la protéine $v$-sis est transformante car elle agit comme le facteur de croissance PDGF. In vivo, le SSV transforme des cellules qui ont le récepteur au PDGF; les cellules transformées produisent un facteur de croissance qui se lie au récepteur du PDGF et se faisant l'active, entraînant ainsi la production constitutive, c'est-à-dire permanente et auto-entretenue, d'un signal de croissance.

Ce mécanisme de croissance qu'on appelle autocrine (par opposition au mécanisme paracrine où la cellule est stimulée par un facteur de croissance produit par une autre cellule) peut agir non seulement dans les cellules transformées mais également, dans certaines conditions, lors de la croissance cellulaire normale : I'analyse de l'expression du proto-oncogène $\mathrm{c}$-sis au cours du développement suggère que le PDGF pourrait stimuler la croissance des cellules cytotrophoblastiques du placenta humain durant une phase précise de l'embryogénèse.

\section{Oncogènes et récepteurs à des facteurs de croissance.}

L'étude de l'oncogène $v$-erbB du virus de l'érythroblastose aviaire (AEV) a fourni la preuve que des oncogènes pouvaient également coder pour des récepteurs à des facteurs de croissance. Les rétrovirus contenant le gène v-erbB se répliquent dans différents types cellulaires de la moelle osseuse mais transforment sélectivement les cellules souches de la lignée rouge, provoquant ainsi une croissance non contrôlée d'érythroblastes immatures. Ces derniers peuvent néanmoins se différencier en globules rouges matures, mais la maturation ne nécessite pas la présence d'érythropoiétine. Ceci suggère que les voies de transmission du signal normalement utilisées par les récepteurs spécifiques de la lignée rouge, telle que l'érythropoiétine, sont constitutivement activées par le produit de l'oncogène $v$-erbB. La surprise fut de constater que ce dernier, la protéine GP74 $\mathrm{v}^{\mathrm{erbB}}$, est une version modifiée du récepteur à l'EGF (Downward et al., 1984). Comme nous l'avons vu plus haut, ce récepteur a une activité 
tyrosine kinase. Bien qu'elle ne possède qu'une très courte région extracellulaire et que sa partie intracytoplasmique soit légèrement tronquée, la GP74 v $v^{\text {erbB }}$ conserve néanmoins l'activité tyrosine-kinase et la capacité de s'auto-phosphoryler. Comment ces modifications peuvent-elles provoquer l'activité oncogénique du récepteur? Comment se fait-il que I'AEV transforme des cellules qui ne possèdent pas de récepteurs à l'EGF ? Ces questions sont encore sans réponses, mais les études de différents mutants de la protéine v-erbB démontrent que des modifications même infimes du récepteur peuvent directement contribuer à la transformation.

L'analyse d'un autre oncogène, le gène $v$-fms du virus sarcomateux félin $\mathrm{SM}-\mathrm{FeSV}$, et de sa contrepartie cellulaire le gène $c$-fms, confirme l'hypothèse que des oncogènes peuvent être des homologues de récepteurs à des facteurs de croissance : elle montre que le récepteur au facteur de croissance CSF-1 (colony stimulating factor 1 ) est le produit du gène $c$-fms (Sherr et al., 1985). Ce dernier est immunoprécipitable par des anticorps dirigés contre la protéine $v$-fms. Contrairement à la protéine $v$-erbB qui est incapable de fixer l'EGF, la glycoprotéine $v$ - $\mathrm{fms}$, dont la partie extra-cellulaire n'est pas tronquée, lie le facteur de croissance CSF. Cette fixation pourrait provoquer la stimulation continue (par un mécanisme de croissance autocrine) des cellules infectées par le SM-FeSV, conduisant ainsi à leur transformation.

\section{Oncogènes et signaux de transduction.}

Une fois que le récepteur au facteur de croissance a été activé par sa liaison avec le ligand, le signal mitotique doit voyager du cytoplasme au noyau pour permettre la synthèse d'ADN et la division cellulaire. Les différentes étapes de ce voyage ne sont pas encore connues. II semble cependant qu'il existe plusieurs voies de transduction du signal qui font intervenir des protéines transductrices du signal, des effecteurs et des " seconds messagers", qui servent en quelque sorte de relai d'information. S'il n'est pas encore possible de décrire la chronologie des événements qui aboutissent à l'arrivée du signal au noyau, on connaît néanmoins des mécanismes et des composants cellulaires qui jouent indéniablement un rôle majeur dans la transduction du message. Un de ces mécanismes est la phosphorylation qui permet de moduler l'activité de protéines d'une façon rapide et réversible. Le fait que de nombreux récepteurs aux facteurs de croissance -EGF, PDGF, CSF-1, insuline et IGF-1 (insuline like growth factor)- et plusieurs produits d'oncogènes ( $v$-erbB, $v$-fms, neu, $v$-abl...) possèdent une activité tyrosine-kinase et peuvent également s'auto-phosphoryler laisse supposer que la phosphorylation joue un rôle important dans la réponse mitogénique. Mais les kinases agissent de façon pléiotropique, c'est-à-dire qu'elles phosphorysent un grand nombre de protéines cellulaires, et on ne sait pas encore, en dépit de nombreux travaux, quels sont les substrats critiques pour la transformation.

L'étude de l'action des tyrosine-kinases sur l'hydrolyse d'un composant lipidique mineur de la membrane montre à quel point la phosphorylation est un processus complexe qui engendre une cascade d'événements qui sont essentiels à la transmission du signal: on a récemment découvert que certains facteurs de 
croissance et certains oncogènes, en particulier ceux qui ont une fonction tyrosine-kinase (tel $c$-src), stimulent le métabolisme des phosphatidylinositols (PI) dans la membrane entraînant la production d'inositol triphosphate (IP3) et de diacylglycérol (DG) (voir fig. 2). Ces messagers secondaires transmettent à leur tour l'information à l'intérieur de la cellule : I'IP3 entraîne le relargage du $\mathrm{Ca}^{+}$ du réticulum endoplasmique; le $\mathrm{Ca}^{+}$peut lui-même agir comme messager secondaire dans la mesure où une augmentation de sa concentration intracellulaire permet d'activer la transcription d'un certain nombre de gènes dont les proto-oncogènes $\mathrm{c}$-myc et $\mathrm{c}$-fos. Le DG stimule les sérine- et les thréonine-kinases, en particulier la protéine kinase $C(P K C)$. Cette dernière active l'échange $\mathrm{Na}^{+} / \mathrm{H}^{+}$, qui à son tour entraîne une augmentation du $\mathrm{pH}$ intracellulaire. Cette augmentation stimule également la transcription de certains gènes, dont les gènes $\mathrm{c}$-myc et $\mathrm{c}$-fos.

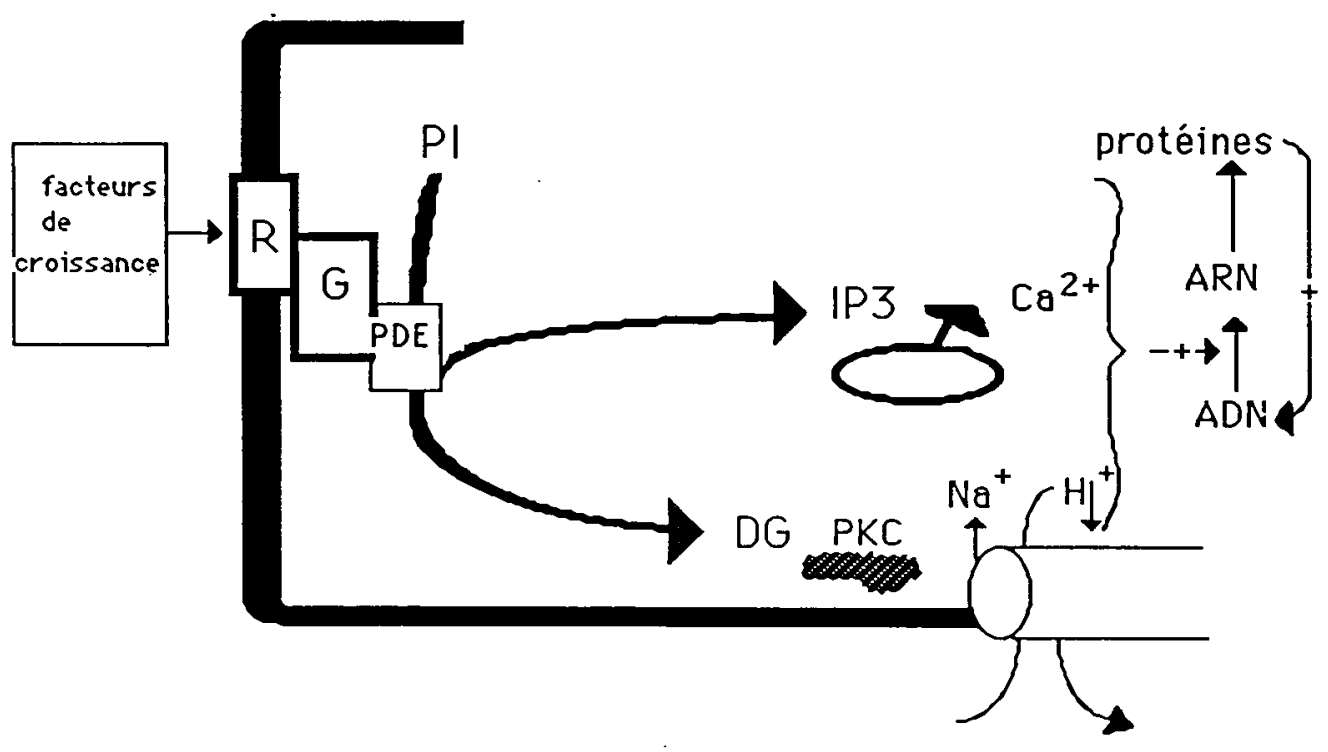

FIG. 2. - Voie de transduction du signal par l'intermédiaire du phosphatidylinositol (PI). Un récepteur $R$ est spécifiquement activé par un facteur de croissance. Une des $G$ protéines couple ce récepteur à la phosphodiestérase (PDE) qui hydrolyse le PI en inositol triphosphate (IP3) et diacylglycerol (DG). Le DG, par l'intermédiaire de la protéine kinase $C$ (PKC) active l'échange $\mathrm{Na}^{+} / \mathrm{H}^{+}$, ce qui augmente le pH intracellulaire. L'IP3 mobilise le calcium intracellulaire. L'augmentation du pH et du $\mathrm{Ca}^{+}$contribue à l'activation de la transcription et à la synthèse de protéines qui à leur tour provoquent la synthèse d'ADN (d'après Berridge, 1986).

Une classe bien connue de molécules à fonction transductrice du signal est la famille des $\mathrm{G}$ protéines. Ces protéines, associées à la membrane, transmettent les signaux extra-cellulaires à des systèmes effecteurs variés, incluant l'adénylate 
cyclase. L'interaction des $\mathrm{G}$ protéines avec les enzymes effecteurs est gouvernée non seulement par les récepteurs aux hormones ou aux facteurs de croissance mais également par le GTP (guanosine triphosphate). La liaison au GTP permet aux $G$ protéines d'interagir avec les effecteurs appropriés en libérant du GDP. On a découvert qu'il existe une homologie de séquences entre une des sous-unités de protéines $\mathrm{G}$ et les proto-oncogènes de la famille ras. Ces proto-oncogènes sont très conservés dans le règne animal. II existe chez les mammifères trois gènes fonctionnels étroitement liés, $c$-Ha-ras, $c$-Ki-ras et $N$-ras, qui codent pour les protéines $\mathrm{p} 21^{\text {ras }}$. Tout comme les protéines $\mathrm{G}$, les protéines $\mathrm{c}$-ras normales sont associées à la membrane plasmique (cette localisation est indispensable à leur fonction); elles se lient aux GTP et ont une activité GTPase. Du fait de cette analogie fonctionnelle, les protéines ras pourraient également participer à la transduction des signaux mitotiques. La forme ras transformante est codée par un oncogène présent dans différentes souches de virus murins sarcomateux et dans une grande variété de tumeurs humaines. Les protéines transformantes ras se lient au GTP mais l'hydrolysent très peu. On ne sait pas actuellement comment les mutations qui caractérisent les protéines ras modifiées confèrent aux p $21^{\text {ras }}$ une activité transformante.

\section{Oncogènes et facteurs de transcription.}

Comment les facteurs de croissance gouvernent-ils la prolifération et/ou la différenciation? II s'écoule un long laps de temps entre l'arrivée du stimulus à la surface de la cellule et la synthèse d'ADN. Que se passe-t-il à l'intérieur du noyau ? La régulation de l'expression des gènes s'effectue grâce à l'interaction de facteurs régulateurs agissant de façon positive (activateurs) ou négative (inhibiteurs) avec des multiples éléments ou séquences de l'ADN (promoteurs et/ou enhancers) généralement localisés en 5' des séquences codantes. Ces facteurs de transcription appartiennent à un réseau de protéines nucléaires dont l'expression conjointe ou successive permet la reprogrammation de l'expression génétique en réponse à des signaux extracellulaires.

Dans les minutes qui suivent la stimulation cellulaire, on observe un pic de synthèse d'ARN qui se traduit par l'apparition d'au moins cent nouveaux transcrits dont les transcrits des proto-oncogènes $c$-myc et $c$-fos. II y a quelques années l'hypothèse suivante avait été formulée: les protéines $c$-myc et $c$-fos nouvellement synthétisées seraient capables d'activer sélectivement la transcription de certains gènes en se fixant spécifiquement à leurs séquences régulatrices. Les travaux entrepris sur la protéine $\mathrm{c}$-fos et les protéines qui lui sont associées confirment cette hypothèse, tout en montrant que le mécanisme de fixation et d'activation transcriptionnelle est un peu plus complexe : en fait la protéine c-fos ne se lie pas directement de façon spécifique à l'ADN. Elle le fait par l'intermédiaire d'une liaison à une autre protéine qu'on appelle la protéine AP1, pour activator protein 1. Cette protéine (initialement purifiée à partir d'extraits nucléaires de la cellule humaine Hela) active, comme son nom l'indique, la transcription des gènes qui possèdent dans leurs séquences régulatrices un motif AP1, c'st-à-dire une séquence de nucléotides reconnue spécifiquement par la 
protéine AP1. Ces motifs AP1 se retrouvent dans les séquences régulatrices de gènes variés viraux ou cellulaires. Lorsque le facteur AP1 se lie au motif AP1, il en résulte une augmentation de l'activité transcriptionnelle du gène correspondant (Rauscher et al., 1988).

Un nouvel oncogène, $v$-jun, a récemment été identifié par Vogt et ses collaborateurs au cours de l'analyse d'un virus aviaire sarcomateux nouvellement isolé, I'ASV17. Contrairement aux oncogènes précédemment isolés d'autres ASV, la protéine $v$-jun ne possède pas une activité tyrosine-kinase. L'étude de sa séquence en acides aminés a révélé une homologie entre une de ses extrémités (la partie carboxy-terminale) et le domaine qui se lie à l'ADN d'un facteur transcriptionnel de la levure, la protéine GCN4. Cette similarité structurale reflète une relation fonctionnelle : tout comme le facteur GCN4, la protéine v-jun se fixe à I'ADN. Les cellules normales de vertébrés contiennent le gène $c$-jun dont la séquence est très proche du gène $v$-jun et dont ce dernier dérive très probablement. Les travaux tout récents de Tjian, Vogt et collaborateurs ont montré que c-jun codait pour la protéine AP1 (ou une protéine très apparentée) et que la protéine nucléaire virale $v$-jun se fixait également à l'ADN des cellules transformées par l'intermédiaire du même motif AP1 (Bos et al., 1988).

Ces études montrent clairement le rôle que peuvent jouer les oncogènes dans la dérégulation de la transcription, renforçant l'idée que la modification d'éléments régulateurs transcriptionnels peuvent directement entraîner la transformation. Cette notion avait été précédemment acquise en partie grâce aux travaux sur le produit du gène $v$-erbA du virus aviaire. L'oncogène $v$-erbA bloque la différenciation des cellules rouges immatures déjà transformées par un oncogène à activité tyrosine-kinase, tel le produit du gène $v$-erbB. La protéine v-erbA est une forme modifiée du récepteur nucléaire à l'hormone thyroïdienne T3, dont le domaine de liaison avec l'ADN est différent de celui de la protéine cellulaire $c$-erbA. La poursuite de l'analyse de v-jun et v-erbA et de leurs contreparties cellulaires permettra de comprendre comment des perturbations du contrôle transcriptionnel contribuent à l'acquisition d'un phénotype oncogénique et quels sont les processus de régulation qui contrôlent l'expression normale d'un gène.

\section{Oncogènes et développement.}

Du fait de leur implication dans les processus de prolifération et/ou différenciation cellulaire et de leur conservation à travers l'évolution, il était tentant de penser que les oncogènes pouvaient participer aux processus normaux du développement. De nombreux laboratoires se sont lancés dans l'analyse de l'expression des c-onc au cours de l'embryogénèse dans l'embryon et les annexes embryonnaires ainsi que dans les cellules de tératocarcinome (pour une revue, voir Adamson, 1987). Ces études, qui permettent d'établir pour un protooncogène donné un profil d'expression qui lui est caractéristique, ne permettent cependant pas encore d'assigner à un proto-oncogène particulier un rôle précis dans une décision développementale. La preuve ultime en serait d'obtenir des mutants d'un produit c-onc dont l'absence ou la modification aboutirait à un 
développement anormal. Cette tâche est difficile chez les mammifères, mais plus aisée chez la drosophile où l'on connaît un certain nombre de mutants de développement. L'étude du gène wingless et de son homologue murin, l'oncogène int-1, nous rapproche de ce but. C'est le premier exemple d'un oncogène dont l'équivalent muté chez la drosophile correspond à un mutant connu du développement.

L'oncogène int- 1 est un des oncogènes cellulaires activés par l'insertion du virus MMTV (mouse mammary tumor virus) dans les tumeurs mammaires de la souris. Le gène int-1, placé dans un vecteur rétroviral, peut également entraîner la transformation morphologique de cellules épithéliales mammaires en culture. Son expression varie au cours du développement de la souris, où le gène est transcrit dans le système nerveux des embryons âgés de 9 à 14 jours. Chez l'adulte, on la retrouve uniquement dans les spermatides. Chez la drosophile, le gène wing/ess, identifié à l'origine par une mutation qui transforme l'aile en une duplication du notum (partie du thorax), appartient au groupe de mutations appelé «polarité de segment ». Les embryons qui possèdent de telles mutations ont un nombre normal de segments, mais l'espace entre deux segments ne possède pas une structure cuticulaire normale. Rijsewijk et al. (1987) ont montré récemment que le gène wing/ess était l'homologue du gène int-1 de la souris. L'injection d'ARN wingless anti-sens (c'est-à-dire complémentaires de l'ARN et qui en s'appariant avec l'ARN empêche sa traduction) à des embryons de type sauvage produit le phénotype mutant, ce qui démontre que la fonction wingless est bien attribuable au gène wingless (ou Dint-1) (Cabrera et al., 1987). L'homologie entre les protéines wingless et int-1 est si forte qu'il est hautement probable que les gènes remplissent des tâches équivalentes chez la mouche et la souris. Cette fonction doit obligatoirement participer à ce processus fondamental que ces deux animaux si différents utilisent. Il est à parier, vu leurs séquences, que ces molécules sont sécrétées et qu'elles peuvent agir comme des facteurs de croissance, qui, en fonction de la cellule cible, auraient soit un pouvoir de différenciation, soit un pouvoir transformant.

\section{Conclusions.}

Nous avons vu que les oncogènes codaient pour des molécules impliquées dans les processus fondamentaux de la vie cellulaire, la croissance, la prolifération, et la différenciation et qu'ils contribuaient ainsi au développement harmonieux d'un individu. Cependant, si la fonction de certains oncogènes est bien connue, l'activité et le rôle biologique de la majorité d'entre eux restent à élucider, d'autant plus que nous sommes certainement encore loin d'en avoir dressé la liste complète. La voie d'approche entreprise ces dix dernières années qui consiste à étudier les mécanismes qui aboutissent à la transformation maligne dans les cancers induits par des rétrovirus ou par des carcinogènes est d'une importance capitale. En effet, elle permet non seulement d'isoler de nouveaux oncogènes, mais également d'identifier leurs homologues cellulaires et d'envisager le rôle qu'ils peuvent jouer dans des processus biologiques normaux. 
Le développement récent de la «transgénèse » ou fabrication d'animaux transgéniques offre par ailleurs les moyens d'étudier in vivo dans le temps, c'est-à-dire au cours du développement, et dans l'espace, c'est-à-dire dans un organe ou un tissu particulier, la fonction des oncogènes. Les animaux transgéniques sont des animaux qui ont intégré dans toutes les cellules de leur organisme une nouvelle séquence d'ADN introduite expérimentalement durant l'embryogénèse précoce. Ces animaux permettent d'étudier les conséquences biologiques de l'expression anormale de gènes ; expression anormale dans la mesure où, grâce à l'inclusion de séquences régulatrices appropriées et/ou inductibles, elle peut être sélectivement dirigée dans un ou des tissus spécifiques et/ou modulée durant le développement (pour un article récent sur les souris transgéniques et l'oncogenèse, voir Williams et al., 1988). Cette approche pratiquée par de plus en plus de laboratoires devrait permettre d'enrichir considérablement nos connaissances sur les mécanismes moléculaires de l'oncogénèse et de comprendre quels sont les événements multiples qui sont requis pour aboutir à une transformation maligne.

$27^{e}$ Réunion de la Société francaise pour l'Etude de la Fertilité. Paris, 29, 30 sept., $7^{\text {er }}$ oct. 1988.

\section{Références}

Etant donné l'abondance de la littérature concernant les sujets que nous avons abordés, nous ne citerons que les références rencontrées dans le texte auxquelles nous avons ajouté certaines revues récentes qui permettront d'approfondir ces questions:

Oncogènes et contrôle de la croissance :

BERRIDGE M. J., 1986. Inositol lipids and cell proliferation, 147-150. In P. KAHN et T. GRAF, Oncogenes and growth control. Springer-Verlag.

COLLETT M. S., ERICKSON R. L., 1978. Protein kinase activity associated with the avian sarcoma virus src gene products. Proc, nat. Acad. Sci. U.S.A., 75, 2021-2024.

DOOLITTLE R. F., HUNKAPILLER M. W., HOOD L. E., De VARE S. G., ROBBINS K. C., AARONSON S. A., ANTONIADES H. N., 1983. Simian sarcoma virus oncgene, $v$-sis, is derived from the gene (or genes) encoding a platelet derived growth factor. Science, 221, 275-276.

DOWNWARD J., YARDEN Y., MAYES E., SCRACE G., TOTTY N., STOCKWELL P., ULLRICH A., SCHLESSINGER J., WATERFIELD M.D., 1984. Close similarity of epidermal growth factor receptor and $v$-erbB oncogene protein sequences. Nature, 307, 521-527.

HUNTER T., COOPER J. A., 1985. Protein-tyrosine kinases. Annu. Rev. Biochem., 54, 897-930. KRONTIRIS T. G., COOPER G. M., 1981. Transforming activity of human tumor DNAs. Proc. nat. Acad. Sci. U.S.A., 78, $1181-1184$.

MURRAY M. J., SHILO B. Z., SHIH C., COWING D., HSU H. W., WEINBERG R. A., 1981. Three different human tumor cell lines contain different oncogenes. Cell, 25, 355-361.

ROZENGURT E., 1986. Early signals in the mitogenic response. Science, 234, 161-166. 
SHERR C. J., RETTENMIER C. W., SACCA R., ROUSSEL M. F., LOOK A.T., STANLEY E. R., 1985. The $\mathrm{c}$-fms protooncogene product is related to the receptor for the mononuclear phagocyte growth factor, CSF-1. Cell, 41, 665-676.

STEHELIN D., VARMUS H. E., BISHOP J. M., VOGT P. K., 1976. DNA related to the transforming gene(s) of avian sarcoma virus is present in normal avian DNA. Nature, 260, 170-173.

USHIRO H., COHEN S., 1980. Identification of phosphotyrosine as a product of epidermal growth factor-activated protein-kinase in A431 cell membranes. J. biol. Chem., 255, 8363-8365.

WATERFIELD M. D., SCARCE G. J., WHITTLE N., STROOBAND P., JOHSON A., WASTESON A., WESTERMARK B., HELDIN C. H., HUANG J.S., DEUEL T. F., 1983. Platelet-derived growth factor is structurally related to the putative transforming protein $\mathrm{p} 28^{\text {sis }}$ of simian

Revue dans: sarcoma virus. Nature, 304, 35-39.

MERCOLA M., STILES C., 1988. Growth factor superfamilies and mammalian embryogenesis. Development, 102, $451-460$.

MÜLLER R., 1986. Proto-oncogenes and differentiation. Trends Biochem. Sci., 11, 129-132.

et également dans le livre très complet Oncogenes and growth control, édité par P. KAHN et T. GRAF, Springer-Verlag (1986).

\section{Oncogènes et facteurs de transcription :}

BOS T. J., BOHMANN D., TSUCHIE H., TJAN R., VOGT P., $1988 . \quad$-jun encodes a nuclear protein with enhancer binding properties of AP-1. Cell, 52, 705-712.

RAUSCHER III F. J., SAMBUCETTI L. C., CURRAN T., DISTEL R. J., SPIEGELMAN B. M., 1988. Common DNA binding site for Fos protein complexes and transcription factor AP-1. Cell, 52, $471-480$.

Revue dans:

PAWSON T., 1987. Transcription factors as oncogenes. T/G, 3, 333-334.

VARMUS H. E., 1987. Oncogenes and transcriptional control. Science, 238, 1337-1339.

\section{Oncogènes et développement :}

RIJSEWIJK F., SCHUERMANN M., WAGENAAR E., PARREN P., WEIGEL D., NUSSE R., 1987. The drosophilia homolog of the mouse mammary oncogene int-1 is identical to the segment polarity gene wingless. Cell, 50, 649-657.

CABRERA C. V., ALONSO M. C., JOHNSTON P., PHILLIPS R. G., LAWRENCE P. A., 1987. Phenocopies induced with antisense RNA identify the wingless gene. Cell, 50, 659-663.

Revue dans:

ADAMSON E. D., 1987. Oncogenes in development. Development, 99, 449-471.

BENDER W., PELFER M., 1987. Oncogenes take wing. Cell, 50, 519-520.

WILLIAMS R. L., COURTNEIDGE S. A., WAGNER E. F., 1988. Embryonic lethalities and endothelial tumors in chimeric mice expressing polyoma virus middle T oncogene. Cell, 52, 121-131. 